\title{
O QUE SIGNIFICA SER REALISTA SOBRE AS MEDIÇÕES CIENTÍFICAS: UMA TAXONOMIA PARA POSICIONAMENTOS (ANTIR)REALISTAS
}

Félix Flores Pinheiro

\section{RESUMO}

O presente artigo possui por objetivo oferecer uma taxonomia inicial para classificar posicionamentos realistas e antirrealistas acerca das medições científicas. Para tanto, exploramos principalmente duas questões, a primeira, o que significa ser realista sobre as medições científicas; a segunda, tendo em vista esse significado, como podemos classificar diferentes abordagens disponíveis na literatura da área. Observando algumas dificuldades em torno da obscuridade do termo "realismo", efetuamos uma discussão sobre de que modo ele é entendido no contexto das discussões filosóficas sobre as medições científicas. Como resultado dessa discussão, obtemos critérios que permitem construir uma classificação subdivida em três grandes campos: realismos, antirrealismos e "áreas cinzentas".

Palavras-chave: Filosofia da medição. Realismo. Objetividade Científica. Medições científicas.

\begin{abstract}
The present paper aims to provide an initial taxonomy to classify realistic and antirealistic positions on scientific measurements. In order to do so, I mainly explore two questions. The first one is what is to be realistic about scientific measurements. The second, in view of this meaning, how we can classify different approaches available in the area literature. Observing some difficulties surrounding the obscurity of the term "realism", I discuss how it is understood in the context of philosophical discussions of scientific measurements. As a result, we place a criteria that allow us to construct classification subdivided into three main fields: realism, antirealism and "gray zones".
\end{abstract}

\footnotetext{
${ }^{1}$ Mestre em filosofia pela UFSM, doutorando em filosofia na UFSC bolsista CAPES.
} 
Keywords: Philosophy of measurement. Realism. Scientific Objectivity. Scientific measurements.

\section{1 - Introdução}

A filosofia contemporânea possui uma subárea de investigação conhecida por filosofia da medição. ${ }^{2}$ Pondo as medições científicas enquanto principal objeto de estudo, a filosofia da medição investiga uma coleção de problemas que emergem dessa prática, adentrando temas tradicionais na filosofia da ciência, tais como racionalidade, causalidade, objetividade, representação, produção de conhecimento e justificação. De um ponto de vista metodológico, os estudos em filosofia da medição visam esclarecer os elementos epistêmicos, ontológicos, semânticos e tecnológicos que tornam as medições possíveis e adequadas. De um ponto de vista histórico, o marco fundador dessa subárea tem sido atribuído ao trabalho de Helmholtz $(1921)^{3}$ dedicado aos fundamentos epistêmicos das medições científicas, intitulado "Numbering and Measuring from an Epistemological Viewpoint" e publicado originalmente em 1887. ${ }^{4}$ Passado mais de um século desde essa publicação, a filosofia da medição atual é composta por uma variedade de escolas de pensamentos diferentes. ${ }^{5}$ Enquanto alguns desses estudos se autoproclamam abordagens realistas medições científicas, outros são vistos como antirrealistas.

${ }^{2}$ Como ressalva Tal (2017a), como as medições não são práticas exclusivamente científicas, a filosofia da medição atual não é simplesmente uma subárea da filosofia da ciência, mas interdisciplinar (com temas transversais entre a epistemologia, a metafísica, a filosofia da ciência, da linguagem e, sobretudo, da tecnologia), mesmo seu principal escopo de estudo sendo constituído pelas medições científicas.

${ }^{3}$ Citamos aqui a versão traduzida para o inglês por Malcolm Lowe, presente na coletânea "Hermann von Helmholtz Epistemological writings".

${ }^{4}$ Uma síntese do período de formação da filosofia da medição foi elaborada por Díez (1996a) e por Michell (1993, 1999).

${ }^{5}$ Uma síntese dessas escolas pode ser encontrada no verbete elaborado por Tal (2017b). 
Todavia, a utilização desses rótulos pelos autores da área não abarca claramente grande parte dos posicionamentos que encontramos nessa literatura. ${ }^{6}$ Nesse sentido, o presente artigo possui por objetivo principal discutir como devemos encarar alguns desses posicionamentos, se podemos e por quais razões poderíamos classificá-los como posturas realistas ou antirrealistas. Para tanto, nossa investigação atenta para três dificuldades especiais na compreensão do que sejam posturas realistas sobre as medições científicas.

A primeira dificuldade advém do fato de que a discussão em filosofia da ciência sobre o realismo científico (doravante $\mathrm{RC}$ ) se dirige especialmente a posicionamentos com relação às teorias científicas, precisando ser adaptada para levar em conta as especificidades do debate sobre as medições. Esse problema será aqui contornado observando como a própria literatura da filosofia da medição tem se utilizado do termo "realismo". Assim, levaremos em conta algumas das ideias salientadas por alguns autores que afirmaram defender alguma forma de realismo sobre as medições científicas, são eles Van Brakel (1984), Byerly e Lazara (1973), Michell (1993, 1999, 2004, 2005), Swoyer (1987) e Teller $(2012,2013)$. A segunda e - do nosso ponto de vista a principal - dificuldade que uma taxonomia para as posicionamentos realistas na filosofia da medição enfrenta, reside no fato de que abordagens autoproclamadas (e classificadas por outros como) realistas

\footnotetext{
${ }^{6}$ Apesar da filosofia da medição ser uma área pouco conhecida, esses posicionamentos serão apenas brevemente introduzidos e discutidos no decorrer desse texto. Adaptados aos nossos propósitos, salientaremos aspectos mínimos necessários para a compreensão da discussão que aqui está sendo realizada. Foge ao escopo desse artigo efetuar uma introdução extensiva a cada um deles, bem como avaliá-los e/ou discutir suas fraquezas, visto que isso demandaria análises individuais que exigem espaço e atenção próprios. Assim, caso o leitor não esteja familiarizado com o assunto, haverá notas indicando textos introdutórios e explicativos que podem ser rapidamente consultados a fim de auxiliar a compreensão do assunto aqui tratado.
} 
nem sempre são posições compatíveis entre si, isto é, há diferentes razões que os autores oferecem para motivar a utilização do termo "realismo", não havendo, por consequência, um critério claro (ou coleção de critérios única) disponível para comparar e classificar os posicionamentos na filosofia da medição. Ambas dificuldades podem ser vistas como as principais motivadoras do presente artigo.

Por fim, uma terceira dificuldade reside no fato de que não há unanimidade para aquilo que se entende por "medição". ${ }^{7}$ No decorrer da história, os autores da área definiram (e/ou caracterizam informalmente) o que é medir de diferentes modos e, como resultado disso, adotar sem a devida análise um ou outro critério já disponível sobre o que significa ser realista nesse contexto pode acarretar em adotar uma visão específica sobre o que sejam as próprias medições. Nesse sentido, parece adequado fugir de uma classificação com base em critérios oferecidos por uma única vertente, from inside, visando encontrar um lugar minimamente comum que permita comparar as diferentes abordagens na literatura, isto é, from above. ${ }^{8}$ Tal lugar deve ser capaz de fornecer inicialmente um esclarecimento sobre o que significa ser realista no contexto da filosofia da medição e, se possível, oferecer critérios para formarmos uma taxonomia de pelo menos alguns dos posicionamentos atualmente disponíveis na literatura da área. Diante disso, o presente artigo desenvolve a hipótese de que esse lugar

\footnotetext{
${ }^{7}$ As concepções sobre o que é "medir" variaram no decorrer da história da filosofia e atualmente há diferentes escolas de pensamento que definem as medições, formal e informalmente, de acordo com seus propósitos e pressupostos próprios (cf. Tal, 2017b).

${ }^{8}$ De modo algo isso pode ser entendido como uma espécie de visão privilegiada ou a partir de lugar nenhum. Ela é uma perspectiva que carrega uma intuição do que sejam as medições, construída de acordo com um propósito bem definido: estabelecer um terreno comum para identificar posicionamentos filosóficos em teorias divergentes entre si, respeitando os pressupostos metafísicos dessas.
} 
pode ser encontrado em uma visão intuitiva e processual das medições, utilizando como critério para classificar posições exatamente essa terceira dificuldade. Uma vez que há diferentes concepções sobre o que seja "medir", essas estão relacionadas com as teses que os autores tecem sobre o que garante a objetividade das medições científicas. Não obstante, há uma estreita conexão entre o tema da objetividade científica e a discussão do RC. ${ }^{9}$ Estressando essa conexão de um ponto de vista dos estudos em filosofia da medição, obtemos não só uma caracterização clara do que seja a tese realista sobre as medições científicas, mas também antirrealista por sua negação e, sobretudo, uma taxonomia entre posicionamentos cuja complexidade não permite um enquadramento simples em ambos rótulos, formando uma "área cinza".

\section{2 - O que significa ser realista sobre as medições científicas?}

Tradicionalmente, as medições foram entendidas como processos de atribuição numérica ao mundo. Todavia, essa caracterização vaga adquiriu significados mais precisos no interior das obras dos diferentes autores. ${ }^{10}$ Por exemplo, enquanto para Stevens (1946) a ideia de atribuição numérica se dirige aos objetos e aos eventos, para Campbell (1920) seu significado é o de representar qualidades através de números e para Michell $(2004,2005)$ consiste na descoberta de atributos/propriedades que os objetos (concretos) possuem. Não obstante, outros autores discordam desse entendimento tradicional e oferecem

\footnotetext{
${ }^{9}$ A conexão entre o que podemos entender por realismo (em diferentes áreas da filosofia) e o tema da objetividade é trabalhada em Dunaway (2018). Como o autor mostra, há diversas formas dessa conexão ser afirmada como trivial. Tentaremos evitar trivializar essa conexão esclarecendo como a literatura da área tem pensado sobre a objetividade das medições científicas.

10 Veja-se Kyburg, 1997.
} 
outras concepções sobre o que é medir, desde obter um output em um sistema informacional (Finkelstein, 1977), até realizar uma inferência em um modelo científico (Tal, 2013). ${ }^{11} \quad$ Essa variedade conceitual tem tornado especialmente complexo qualquer trabalho que vise comparar os posicionamentos disponíveis na filosofia da medição, visto que quaisquer comparações nessa área só fazem sentido à luz de um entendimento sobre o que seja medir. Tal complexidade tem origem no fato de que recorrer a um ou outro entendimento específico requer uma escolha, que precisa ser justificada e que sem o devido cuidado pode comprometer a investigação, resultando em uma análise (a partir da perspectiva da teoria adotada, from inside) que privilegia posicionamentos semelhantes e negligencia teses opostas.

Todavia, como as medições constituem práticas científicas e cotidianas variadas, um viés intuitivo sobre o que elas sejam, observando os elementos presentes nessas práticas, pode ser visto como um ponto de partida para a análise filosófica. ${ }^{12}$ De um ponto de vista intuitivo, medir é uma atividade com um objetivo pré-estabelecido, com alvos pré-determinados, consistindo na realização de uma avaliação comparativa. Mesmo simples, essa caracterização das medições nos auxilia a esclarecer algumas considerações importantes sobre o que elas sejam e nos permitem elucidar seus componentes, resultando em um esquema processual e intuitivo. ${ }^{13}$ Em primeiro lugar, como é

\footnotetext{
${ }^{11}$ Um compilado dessas concepções pode ser encontrado no verbete de Tal (2017b).

$12 \mathrm{O}$ ponto de partida, nesse caso, advém de um senso comum crítico, de modo semelhante ao discutido por Haack (1995). Seus propósitos precisam ser bem definidos e o mesmo só faz sentido enquanto elemento introdutório para uma investigação. Isso significa que ele é provisório, não pode ser visto como um "ponto de chegada", não pode ser exaustivo, tampouco ter a pretensão de esgotar a análise.

13 O esquema que aqui será apresentado consiste em uma síntese de distinções e esclarecimentos distintintos efetuados por Berka (1982)
} 
afirmado por Van Brakel (1984), as medições só podem ser entendidas tendo em vista o contexto dos alvos e objetivos que orientam previamente essa atividade. ${ }^{14}$ Esses alvos são comumente referidos na literatura da área através dos termos "grandezas" e "magnitudes". Uma grandeza pode ser entendida como uma característica mensurável, como por exemplo, a temperatura e o comprimento, sendo também referida por "conceito quantitativo". ${ }^{15}$ Porém, os alvos das medições não são grandezas em um sentido geral, mas o nível de uma manifestação particular, por exemplo, o nível do comprimento da mesa que apoia meu computador, o que é chamado de "magnitude". Se ainda não estiver claro: o alvo de uma medição é a magnitude de uma grandeza (isto é, o nível de alguma coisa manifestada em um objeto ou evento). Portanto, o objetivo de uma medição consiste em avaliar magnitudes.

Essa avaliação consiste em um procedimento de comparação que exige tomar uma unidade padrão de comparação das magnitudes de uma grandeza. Por exemplo, para medir o comprimento da minha mesa, posso utilizar o comprimento de um livro, obtendo como resultado uma comparação entre ambas magnitudes que permita estabelecer uma relação de maior, menor ou semelhante

com a discussão realizada por Frigerio, Giordani \& Mari (2010).

${ }^{14}$ No caso das medições, percebê-las como não sendo livres de contexto não é incompatível com as formas de realismo, visto que as grandezas em alvo são diferentes umas das outras, o que implica apenas constatar que, por exemplo, medir a velocidade envolve um contexto diferente de medir a pressão atmosférica, ou a inteligência, com procedimentos, unidades, escalas e outros elementos próprios para cada caso.

${ }^{15}$ O que é essa característica que possui um nível que é medido, ou seja, o que são as grandezas, é um tema de natureza metafísica cujo refinamento extrapola os propósitos desse artigo. Na literatura da área, ela é caracterizada de vários modos, como uma qualidade, uma propriedade, um atributo, podendo ser vista como algo que objetos exibem, manifestam ou possuem. A fim de respeitar como as diferentes abordagens definem as grandezas e seus pressupostos metafísicos (e por falta de um termo melhor), nos referimos a elas aqui pelo termo "característica". 
entre seus comprimentos. Todavia, os procedimentos de medida científicos são muito mais complexos e refinados do que isso e realizados através de instrumentos cuja construção e correta manipulação exige um background teórico. Isso é facilmente percebido ao constatarmos que só poderíamos medir a massa de algum objeto se possuirmos de antemão algum entendimento sobre o que seja "massa", o qual pode consistir na compreensão de conceitos elaborados pelas teorias científicas e/ou filosóficas sobre o assunto. ${ }^{16}$ Por fim, dispondo de um conhecimento prévio sobre o que queremos medir, de um aparato instrumental empírico para o procedimento de medição, ${ }^{17}$ e de uma unidade padrão para comparar as magnitudes da mesma grandeza em diferentes objetos/eventos, executamos uma medição e obtemos um resultado: a avaliação da magnitude em questão. Usualmente esse resultado é colocado nos termos de um valor numérico acrescido da unidade de referência, o que no exemplo acima pode ser aproximadamente "6 livro-padrão" ${ }^{18}$ Mais ainda, utilizando o comprimento desse livro como unidade padrão para medir outros objetos da minha sala, podemos construir uma classificação entre os maiores e os menores, objetos que medem aproximadamente 2 livro-padrão, 3 livro-padrão, etc., construindo uma "escala" e, se possível, estabelecendo

${ }^{16}$ Essa afirmação é conhecida por problema de coordenação. Resumidamente, o problema de coordenação emerge da pergunta sobre quando e se um procedimento de medida mede aquilo que ele se propõe. Ele consiste na fundamentação da relação entre, por um lado, o que conta como modo legitimo de medição de algo e, por outro, o que é esse algo, salientando que devemos evitar um círculo vicioso (veja-se Cartwright e Chang, 2013, p. 367).

17 A partir daqui o termo "procedimento" se refere aos instrumentos de medida, para distinguir uma parte empírica do esquema geral que formulamos, o qual será referido pelo termo "processo". Assim, um procedimento é um elemento empírico constitutivo do processo de medição, o qual envolve outros elementos, como conceitos e escalas.

${ }^{18}$ Alguns autores têm argumentado que é perfeitamente razoável pensar os resultados das medições fora de um âmbito numérico, como por exemplo Roberts (1979) e Tal (2013). Porém, não abarcaremos tais casos aqui. 
relações proporcionais entre eles (por exemplo, da minha mesa ser aproximadamente 2 vezes mais comprida que minha cadeira). ${ }^{19}$ Vale ressaltar que é por conta da utilização desse valor numérico que as medições foram vistas, como já dissemos, enquanto maneiras de atribuir números ao mundo.

Apesar de intuitiva e informal, essa elucidação sobre o funcionamento geral das medições esclarece seus componentes e salienta algumas relações entre eles, resultando em uma organização processual simples, de fácil aceitação, como está esquematizado na Figura 1. Poder-seia discutir o que são os resultados das medições, o que são as grandezas, as magnitudes, os procedimentos adequados, como essas coisas estão relacionadas, dentre outros tópicos, mas não faz sentido negar que (1.1) um procedimento físico, (1.2) uma grandeza, (1.3) as magnitudes de uma grandeza, (2.1) um esquema conceitual com (2.2) conceituações sobre a grandeza, (2.3) escalas, (2.4) unidades e (2.5) um resultado, são componentes de nossas práticas de medida.

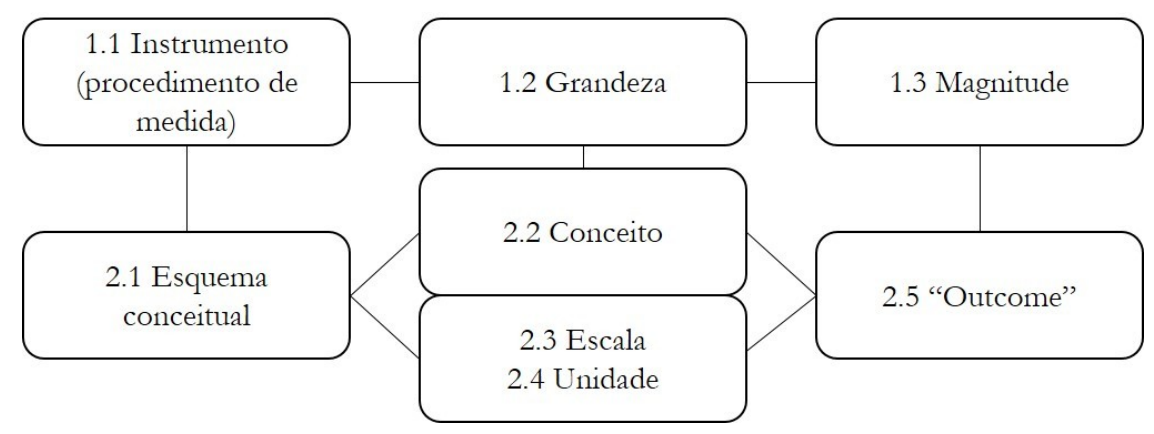

19 O termo "escala" está em aspas pois sua utilização nesse exemplo não é mais que uma visão altamente simplificada e não teórica do que é formar uma escala, nos servindo apenas para propósitos heurísticos. A construção das escalas é um tópico de suma importância na filosofia da medição e que foi trabalhado profundamente por uma das principais teorias da área, a Teoria Representacional da Medição (cf. Díez 1997b). De acordo com ela, utilizando a teoria intuitiva de conjuntos, uma escala é definida como a tripla $\left\langle\mathrm{E}, \mathrm{E}^{\prime}, f\right\rangle$, na qual $\mathrm{E}$ é uma Estrutura de Relações Qualitativas, E' é uma Estrutura de Relações Quantitativas (cujas aridades das relações são as mesmas que as das relações em E) e $f$ é uma função homomorfismo (também chamada de função mapeamento) de E em E' (cf. Frigerio, Giordani e Mari, 2010, p. 127). 
Figura 1. Esquema intuitivo e processual para as medições (elaborada pelos autores). ${ }^{20}$

Organizados dessa maneira, separamos dois níveis para os elementos do procedimento de medição, de modo similar ao que é elaborado por Frigerio, Giordani e Mari (2010): (1.1) é experimental; (1.2) e (1.3) são partes empíricas do processo de medida; enquanto (2.1), (2.2), (2.3), (2.4) e (2.5) são contrapartes teóricas das medições científicas. Vale destacar que em nosso esquema, (2.1) consiste tanto no background teórico que nos auxilia na construção, manipulação dos instrumentos e na interpretação dos seus resultados (o qual pode ser formado por modelos, partes de teorias e teorias científicas completas, se o contexto demandar); quanto nas teorias matemáticas sobre a construção das escalas. Assim, as partes (2.2), (2.3), (2.4) e (2.5) tem origem em (2.1), mas aqui são apresentadas separadamente. Os motivos para isso residem no fato de que algumas teorizações tecem teses que focam em um desses elementos, negligenciando os outros; além de atribuírem papéis epistêmicos distintos para elas no processo de justificação dos fundamentos das medições. $^{21}$

Com o auxílio desse esquema inicial, podemos colocar a pergunta sobre o que significa ser realista sobre as medições científicas utilizando algumas considerações da filosofia da medição. Um dos problemas fundamentais discutidos nessa área nasce ao percebermos que há elementos convencionais nesse processo, acarretando na pergunta sobre até que ponto esses são determinantes e comprometem os resultados das medições científicas. Por

\footnotetext{
${ }^{20}$ Nesse diagrama, os traços entre as partes representam relações que mais adiante serão esclarecidas.

${ }^{21}$ O exemplo paradigmático aqui advém da Teoria Representacional da Medição, a qual nos fornece um corpo teórico detalhado sobre a construção das escalas, sem tocar na questão da construção dos instrumentos de medida e dos conceitos científicos.
} 
exemplo, para medir a magnitude da temperatura de um líquido, podemos escolher que procedimento usar (como um termômetro a gás ou com base no deslocamento do mercúrio), escolher uma escala (digamos, Celsius ou Fahrenheit) e a unidade (relativa a escala escolhida) ${ }^{22}$; por consequência, é possível obter resultados (pelo menos aparentemente) diferentes.

Voltando a atenção para a presença dessas convenções, filósofos questionaram onde elas iniciam e onde elas param, se há algo que não é passível de escolha e o que é esse algo. Questionamentos semelhantes podem ser colocados por outros vieses: (epistêmico) entendendo as medições como um procedimento de avaliação, questionamos o que distingue esse procedimento de uma avaliação subjetiva; ${ }^{23}$ (metodológico) entendendo as medições como um processo, questionamos quais partes dele são determinantes (não acidentais) para os seus resultados; (metafísico) observando as relações entre essas partes, podemos questionar as dependências de umas em relação às outras e a independência delas de nós. Do nosso ponto de vista, tais perguntas foram teorizadas na literatura da área originando constructos teóricos de diferentes vieses com um o mesmo cerne: a garantia da objetividade dos procedimentos de medição. ${ }^{24}$

O termo "objetividade" é utilizado com significados diversos na filosofia da medição, desde o problema colocado

\footnotetext{
${ }^{22}$ No interior da Teoria Representacional da Medição, a unidade é escolhida convencionalmente e antes da construção da escala (cf. Krantz et al, 1971, p. 9).

${ }^{23}$ Isso é realizado por Mari (2003).

${ }^{24}$ É curioso perceber também que medições são vistas enquanto fontes de objetividade (casos paradigmáticos dessa ideia são os escritos de Lorde Kelvin e o ideal de padronização do sistema métrico na revolução francesa). Na atividade científica, parte dessa ideia reside na esperança da eliminação da influência humana nos seus resultados através da utilização de instrumentos e padrões (veja-se Reiss e Sprenger, 2017, seção 4); tese que remonta a um tipo de "objetividade mecânica" (vejase Riordan, p. 42).
} 
por Helmholtz (1921) sobre "o significado objetivo de expressarmos através de números determinados relações entre objetos reais enquanto magnitudes, e sob quais condições podemos fazer isso" (Helmholtz, 1921, p. 75, tradução nossa). Por exemplo, Frigerio, Giordani e Mari (2010) o utilizam no sentido de uma consistência para os resultados (outcomes) entre procedimentos de medida realizados em diferentes ambientes, o que é estendido por Teller $(2012,2013)$ para diferentes instrumentos. Porém, o que significa "objetividade" para as medições científicas foi mais profundamente discutido por Riordan (2015) e, de acordo com a autora, está diretamente relacionado com duas virtudes epistêmicas: a estabilidade e a precisão das medições. Todavia, como nem todas teorias filosóficas sobre as medições dão devida atenção às performances experimentais das medições científicas, ${ }^{25}$ entenderemos por "objetividade" uma caracterização mais ampla e adaptável a essas posições. Seguindo apenas um componente da definição apresentada por Mari et al (2017) e parte do entendimento elaborado por Tal (2017a) para o termo, entenderemos a partir daqui que a objetividade das medições científicas se refere aquilo que, mesmo sempre havendo uma sensibilidade ao contexto nesses procedimentos, evita que essa sensibilidade comprometa seus resultados. Entender aqui o significado de "objetividade" utilizando um "aquilo que" vago é uma lacuna proposital, ela será preenchida pela abordagem filosófica que estivermos tratando. ${ }^{26}$

\footnotetext{
${ }^{25}$ Ocorre que grande parte desses estudos ocorreram antes do que ficou conhecido como empirical turn nas filosofias da ciência e da tecnologia.

${ }^{26}$ Ou seja, dizer que uma medição é objetiva significa dizer que seus resultados não foram comprometidos pelo seu contexto (pelo contrário, como há uma dependência do contexto, ele é condição de possibilidade para os procedimentos). Porém, determinar quando e por quais razões os resultados não foram comprometidos pelo contexto só é possível teorizando sobre o assunto, ou utilizando alguma das teorias
} 
Diante disso, de modo similar ao que ocorre no RC, um realismo sobre as medições científicas está voltado para o produto da atividade científica (que naquele caso são as teorias, mas nesse caso são os resultados das medições), afirmando a conexão desse produto teórico com a natureza, isto é, afirmando a relação entre (1.3) e (2.5). Por conta dessa conexão, para o realista com respeito às medições científicas, as medições não são um simples processo, mas um procedimento que acessa uma realidade - sendo o sucesso desse acesso o que garante a objetividade das medições científicas. Essa ideia é compatível com o que é afirmado por Michell:

A filosofia realista da medição difere de outras [...] de três maneiras. Primeiro, distingue o que é medido de como é medido. Em segundo lugar, sustenta que o que é medido são atributos das coisas, e não as próprias coisas. Terceiro, afirma que, na medição, os números são descobertos em vez de atribuídos (Michell, 2004, p. 3, tradução nossa).

Esses tópicos sintetizam as próprias teses do autor, que se autoproclama realista sobre as medições. De acordo com ele, magnitudes são partes de relações proporcionais e, por esse motivo, não medimos "coisas" - objetos ou eventos, mas seus atributos: possuir uma propriedade e estar em uma relação de proporção. Nesse sentido, os resultados das nossas medições, os (2.5) outcomes, constituem as partes das relações proporcionais que são descobertas pelo procedimento de medida (e, vale notar, não são redutíveis a eles).

Certamente, a postura de Michell $(2004,2005)$ pode ser entendida como realista, porém, nos parece demasiado

disponíveis, com critérios from inside. Por esse motivo, apesar de, do nosso ponto de vista, as diferentes teorias não estarem em pé de igualdade sobre esse assunto, possuindo umas argumentos mais fortes do que outras, não avaliaremos e respeitaremos o entendimento dos autores, utilizando-os, no que segue, justamente para caracterizar as posições como (antir)realistas. 
Perspectiva Filosófica, vol. 45, n. 1, 2018

exigente pensar que apenas posicionamentos que concordem com a sua terceira asserção sejam entendidas como formas de realismo sobre as medições científicas. Michell $(2004$, 2005) parece identificar a referência das "magnitudes" com os outcomes numéricos de uma medição, o que não é uma condição sine qua non da objetividade das medições, pois o resultado de uma medição pode ser visto como uma representação numérica, ou ainda, como possuindo uma referência não numérica (empírica ou de outra natureza), sem que haja uma contexto-dependência comprometedora. Como salientado por Tal (2017b), a defesa de Michell $(2004,2005)$ consiste em um realismo com enfoque numérico, definindo magnitude enquanto um número (real) com inspiração no conceito de razão de Euclides. Ela se distingue de outras posturas consideradas realistas (inclusive pelos próprios autores) que não argumentam no mesmo sentido, como a de Byerly e Lazara (1973), a de Swoyer (1987) e a de Mundy (1987), por conta desses conceituarem os alvos das medições apenas como propriedades (e não como números). Nesse sentido, as grandezas são entendidas como propriedades objetivas, isto é, cuja existência é independente dos procedimentos de medida e de nossas convenções, dotadas de estrutura. Assim, se há algo que descobrimos efetivamente no procedimento de medida são essas estruturas e são elas que permitem (e supostamente explicam) nossas representações numéricas adequadas, enquanto as magnitudes, por sua vez, são partes constitutivas dessa estrutura que serão representadas numericamente, não descobertas.

De modo similar ao que Chakravartty (2017) afirma na discussão sobre o RC, apesar das variadas formulações para o termo "realismo", podemos encontrar um núcleo 
comum nas ideias de alguns autores com relação aos produtos das medições científicas, em especial quando as observamos a partir do esquema da Figura 1. Esse núcleo comum consiste na afirmação de que uma parte específica do processo de medição é responsável por garantir a sua objetividade, os seus alvos (as partes (1.2) e (1.3)), acarretando em uma visão fundacionalista dos processos de medida, ilustrada na Figura 2. Nesse sentido, ser realista sobre as medições científicas consiste em defender a seguinte tese:

Realismo das Medições Científicas (doravante RMC): os alvos das medições são independentes de nós e dos procedimentos de medida e determinam os seus resultados.

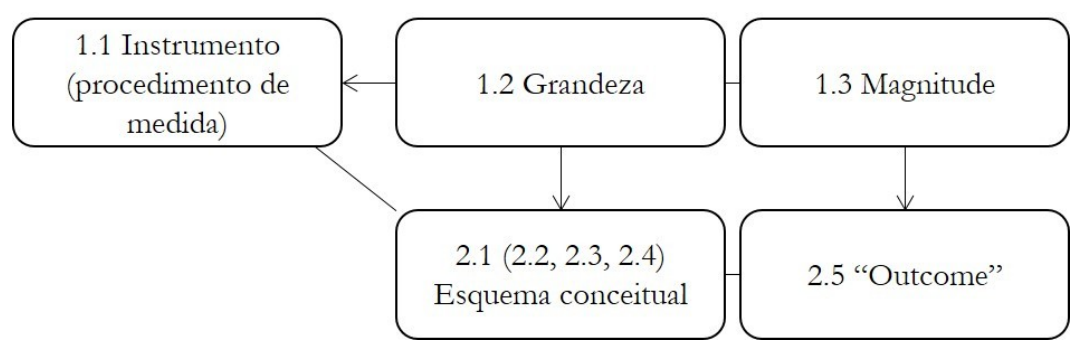

Figura 2. Processo fundacionalista do RCM (elaborado pelos autores). ${ }^{27}$

Novamente, a vagueza proposital dos termos "independentes" e "determinam" visa capturar correspondências que preencham seus significados a partir de diferentes tipos de posicionamentos. É justamente essa vagueza que nos permitirá formular uma taxonomia para (antir)realismos, a qual já pode ser vislumbrada se observarmos cuidadosamente os argumentos que conduzem à tese do RMC. Como Chakravartty (2017) e Michell (2005) afirmam, a discussão em filosofia da ciência sobre o RC possui dimensões metafísicas, epistêmicas e semânticas, sendo sua formulação semântica a tese usualmente defendida. Do nosso ponto de vista, na filosofia da medição

${ }^{27}$ Nesse caso, as flechas indicam as determinações, enquanto os traços não dirigidos representam que há uma relação de dependência mútua. 
Perspectiva Filosófica, vol. 45, n. 1, 2018

ocorre algo semelhante, originando argumentos que sustentam a tese do RMC através de diferentes entendimentos sobre em virtude do que os alvos das medições são independentes dos procedimentos de medida. ${ }^{28}$ Nesse contexto, a dimensão usualmente utilizada também é a semântica, encontrada em grande parte das aparições do termo "realismo" na literatura. Citando exemplos, Van Brakel conceitualiza o realismo enquanto "uma visão sobre uma certa classe de afirmações - por exemplo, declarações relatando os resultados das medições. É uma tese semântica" (Van Brakel, 1984, p. 49, tradução nossa); enquanto Byerly e Lazara afirmam que "uma abordagem realista das medições está comprometida com a visão de que conceitos quantitativos possuem referentes" (1973, p. 27, tradução nossa). Já Cartwright e Chang (2013) opõem o realismo a um nominalismo sobre as medições científicas, afirmando que o último "trata os métodos de medição como definidores de conceito. O outro (...) enquanto métodos para descobrir quantidades objetivas que podemos identificar independentemente da medição" (2013, p. 368, tradução nossa).

A partir dessas intuições, podemos separar claramente o realismo de Michell (2005) das defesas do RMC, pois de acordo com o autor:

Compreender as medições sob o guarda-chuva do conceito realista de verdade nos compromete não apenas com a existência logicamente independente das coisas no espaço e no tempo,

\footnotetext{
${ }^{28}$ Enquanto a dimensão epistêmica do RMC o interpreta enquanto um processo de produção de conhecimento válido, a dimensão metafísica trabalha com suas independências ontológicas. Certamente, há muito mais a ser dito sobre esses pontos, em especial explorando como elas se relacionam entre si. Por exemplo, o viés epistêmico pode argumentar que os resultados das medições constituem conhecimento por conta de aspectos metafísicos e ou semânticos que asseguram a objetividade dos processos de medição. Porém, dada a complexidade dessas relações, não aprofundaremos esses temas nesse momento, elas demandariam um espaço que não dispomos aqui.
} 
mas também com a existência de propriedades e relações estruturadas quantitativamente e com a existência de números reais, entendidos como relações de razão entre níveis específicos desses atributos (Michell, 2005, p. 287, tradução nossa).

Enquanto o RMC afirma que os conceitos quantitativos possuem referentes - e esses geralmente são vistos como propriedades - a tese defendida por Michell (2005) possui um elemento metafísico adicional, que segundo o próprio autor é derivado dos comprometimentos lógicos e ontológicos evidenciados no decorrer de sua abordagem. Sua tese pode ser entendida como: RMC + "que números reais são relações localizadas espaço temporalmente" (Michell, 2005, p. 287, tradução nossa). A presença desse aspecto adicional nos permite não apenas distinguir entre uma forma geral de realismo no contexto das medições científicas e uma mais específica, mas conjecturar outros tipos de realismos nessa discussão, por analogia com os posicionamentos no debate sobre o RC. Esses outros tipos podem ser entendidos na forma de uma "RMC + $\mathrm{x}$ ", na qual $\mathrm{x}$ é uma cláusula voltada para comprometimentos ontológicos. Com essa margem aberta, se como no caso de Michell (2005), essa cláusula versar sobre o caráter indispensável dos alvos das medições científicas serem entendidos de uma maneira específica, em especial nos termos de um comprometimento ontológico; podemos encontrar exemplos de posicionamentos por analogia com a discussão do RC: se esses comprometimentos conduzirem à tese de que grandezas sejam, por exemplo, entidades à la Hacking (1982), conjecturamos o RMC de entidades $(\mathrm{RMC}+\mathrm{E})$; se forem capacidades à la Cartwright (1999), conjecturamos o RMC $+\mathrm{C}$, e assim por diante. Por fim, se essa cláusula versar sobre o caráter indispensável de um compromisso 
ontológico no sentido de uma atitude metodológica, enfraquecendo-a, temos uma espécie de realismo metodológico sobre as medições científicas, como o defendido por Van Brakel: "a ontologia da medição deve ser o realismo moderado, parte porque (enquanto um axioma metodológico) ele leva em conta o contexto das medições de modo muito mais realista que posicionamentos operacionalista ou instrumentalistas" (1984, p. 47, tradução nossa).

Ainda assim, tanto o RMC, quanto um "RMC + $\mathrm{x}$ " afirmam a independência dos alvos das medições no sentido de os conceitos em alvo possuírem referências que não são redutíveis aos procedimentos, nem a uma definição puramente convencional. Por oposição, a defesa de que esses conceitos são definidos por procedimentos (conhecida por operacionalismo) e a defesa de que os mesmos são definidos de modo puramente convencional (conhecida por convencionalismo), constituem posicionamentos antirrealistas sobre as medições científicas. ${ }^{29}$ Todavia, apesar de serem uma espécie de paradigma para posturas antirrealistas nesse debate, como há diferentes modos de negar a tese do RMC, elas não podem ser vistas como os únicos casos de antirrealismo.

\section{3 - Antirrealismos e áreas cinzentas}

Posturas antirrealistas podem ser definidas por oposição ao RMC, mas há diferentes maneiras de negar essa tese. A primeira delas consiste em simplesmente recusar a objetividade das medições científicas. Como estressamos a relação entre aquilo que determina os resultados das medições científicas com uma concepção de

${ }^{29}$ Uma introdução a essas abordagens pode ser encontrada na seção 4 do verbete elaborado por Tal (2017b). 
Perspectiva Filosófica, vol. 45, n. 1, 2018

objetividade baseada na sensibilidade ao contexto, adotamos uma postura antirrealista ao afirmarmos que nada garante essa objetividade, isto é, que os resultados das medições são dependentes de contextos particulares. Denominaremos essa tese por antirrealismo radical das medições científicas, doravante ARMC.

A segunda delas consiste em negar apenas que objetividade das medições seja garantida em um processo fundacional, defendendo, por exemplo, que ela não deriva de uma parte mais fundamental, mas da coerência do processo. Denominaremos essa intuição geral por antirrealismo-coerentismo das medições científicas (ACMC). Essa tese é atualmente desenvolvida pelos autores da assim chamada model-based accounts of measurements, cujo principal expoente é Tal (2013, 2017a, 2017b), as quais entendem que a origem da objetividade das medições científicas não está em um componente específico e interno aos seus processos, mas se dá em virtude de como os diferentes elementos estão relacionados entre si, garantindo uma consistência dos resultados.

Por fim, o último modo de recusar o RMC que mencionaremos aqui, consiste em afirmar que há algo que garante a objetividade das medições científicas, o qual é encontrado em um processo fundacionalista, mas que não são os alvos das medições (isto é, não reside nas magnitudes, nem nas grandezas). Denominaremos essa intuição geral de antirrealismo-fundacionalismo das medições científicas (AFMC). No que segue, trataremos especialmente de alguns aspectos controversos sobre o AFMC.

Apesar da abordagem operacionalista ser vista como paradigma de postura antirrealista, dependendo de como a entendemos a mesma não implica diretamente na defesa de 
que as medições científicas não são objetivas. A principal tese do operacionalismo consiste na afirmação de que o procedimento de medida define os conceitos quantitativos. Por exemplo, o conceito de "temperatura" é definido por um procedimento específico de medida da temperatura. As dificuldades que essa abordagem enfrenta são bem conhecidas: justificar em quais condições procedimentos diferentes medem a mesma grandeza e quando sabemos que eles atingiram a magnitude visada ao invés de algum erro ter ocorrido. Porém, uma atualização dessa posição que superasse essas dificuldades poderia reconstruí-la nos termos de uma vertente antirrealista do domínio semântico e compatível com um viés fundacional das medições. Isso ocorre por conta do domínio semântico do RMC, tal como o entendemos, ser a afirmação conjunta do seguinte:

I) Há algo que garante a objetividade das medições científicas;

II) Esse algo é independente dos procedimentos empíricos de medida;

III) Os alvos das medições possuem referências não procedimentais, não subjetivas, nem puramente convencionais;

IV) Os alvos das medições determinam os seus resultados; ${ }^{30}$

V) Os alvos das medições são esse algo.

Como vimos, o ARMC recusa I e, por consequência as outras asserções. Todavia, o cerne do operacionalismo recusa III) e, por consequência, IV) e V). Contudo, a negação de IV) por parte de um viés operacionalista não implica, prima facie, a negação de I), tampouco a afirmação

\footnotetext{
${ }^{30}$ Essa asserção advém da visão das medições enquanto um processo de descoberta.
} 
Perspectiva Filosófica, vol. 45, n. 1, 2018

de que nada determine os resultados das medições. ${ }^{31}$ Todavia, sem superar as dificuldades supracitadas, os processos das medições estão confinados aos contextos particulares, acarretando na não-objetividade do processo, como discutimos na seção anterior.

Utilizando nosso esquema intuitivo e processual das medições, um viés operacionalista se compromete principalmente com a dependência das grandezas em relação aos procedimentos de medida, formando um processo como está ilustrado na Figura 3.

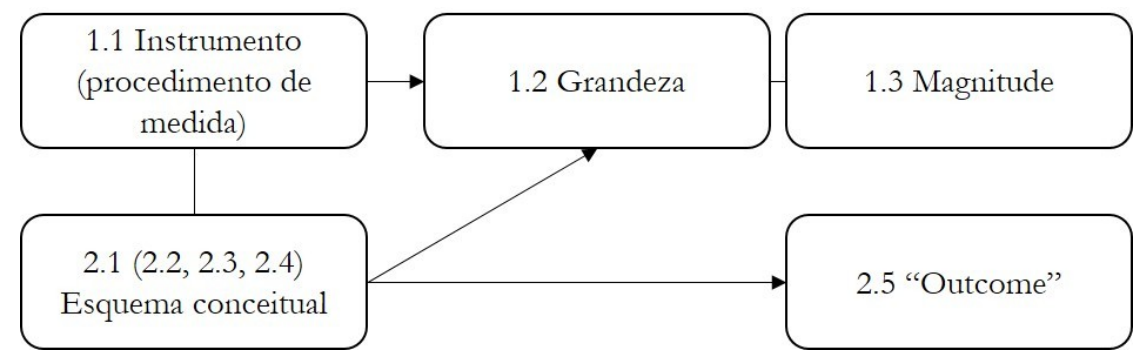

Figura 3. Processo operacionalista

Diante disso, o operacionalismo é uma forma de antirrealismo semântico das medições científicas que não acarreta necessariamente no ARMC. Por outro lado, enquanto essa incompatibilidade depende de como reinterpretarmos e reconstruirmos a visão operacionalista dos processos de medições, mantendo apenas o seu cerne, outras formas de antirrealismo são definitivamente incompatíveis com o ARMC. Por conta dessas abordagens também negarem algumas afirmações do RMC, recusando especialmente as afirmações II) e IV), tais posturas constituem algo como uma "área cinzenta" nessa discussão.

\footnotetext{
${ }^{31}$ Complementando essa afirmação, o fato do operacionalismo em sua formulação clássica, advinda de Bridgman (1927) fracassar em explicar diversos tópicos sobre como as medições são possíveis e adequadas, não torna reformulações futuras da mesma intuição geral automaticamente infrutíferas. Uma possibilidade aqui é reconstruir a posição com respeito a um realismo no domínio epistêmico. Isso pode ser realizado considerando que a construção dos instrumentos pressupõe um (2.1) esquema conceitual, o que pode ser colocado em um processo de refinamento do conhecimento científico através do vínculo entre procedimentos empíricos e representações numéricas.
} 
O ACMC recusa V) por pensar que não há uma parte fundamental no processo de medição e, em sua construção, recusa II) por entender a objetividade nos termos da coerência do processo, o que de algum modo depende também dos procedimentos de medida. Por outro lado, vieses fundacionalistas recusam II) e IV) ao afirmarem que esse fundamento não está nos alvos das medições, mas em algum outro lugar desse processo.

Entender o processo das medições por um viés que não toma os conceitos quantitativos enquanto elementos fundamentais para a sua objetividade tem sido um ponto motivado pela compreensão de que o acesso às magnitudes é relativo ao contexto de medida. Como magnitudes são níveis de grandezas em contextos particulares, mesmo que suponhamos um valor "real", ou "ideal" a ser alcançado, elas não fazem sentido isoladas, adquirindo significados no decorrer de um processo de comparação. Assim, a referência de uma magnitude só pode ser preenchida de significado após o procedimento, ou supondo valores que precisam ser pré-estabelecidos em algum lugar, não fazendo sentido considerá-las fora do contexto de medição (seja ele prático, ou teórico). Do mesmo modo, a validade de um outcome, isto é, quando o processo mediu adequadamente e obteve um resultado apropriado, ${ }^{32}$ só faz sentido à luz de valores pré-estabelecidos como referência, ou na presença de um método de construção de outcomes que não requeira esses valores prévios. Essa última possibilidade é chamada de "medição fundamental" e foi desenvolvida pelas abordagens representacionais das medições; enquanto a primeira é denominada por "medição derivada" e recebe especial atenção dos vieses coerentistas das medições científicas.

${ }^{32}$ Uma defesa realista sobre esse aspecto específico foi discutida por Teller (2013, 2014), denominada por realismo de precisão. 
A abordagem representacionalista das medições científicas questiona os fundamentos epistêmicos das medições fundamentais. ${ }^{33}$ Grosso modo, utilizando a teoria intuitiva de conjuntos como framework, a abordagem representacionalista entende que medir pode ser visto como um procedimento de representação numérica de uma estrutura de relações qualitativas em uma estrutura de relações quantitativas, o que nesse caso significa construir um homomorfismo ${ }^{34}$ entre ambas. Podemos entender os processos de medições através dessa abordagem organizando-os em três etapas. Inicialmente, observamos regularidades empíricas e as representamos através de axiomas que regulamentam o comportamento de relações em uma estrutura de relações qualitativas. Após isso, lançamos estruturas relacionais quantitativas e funções homomorfismo enquanto hipóteses de representação para as estruturas de relações qualitativas. Por fim, provamos teoremas explorando as características matemáticas dessas estruturas, o que nos permite estabelecer quando uma representação numérica é apropriada (isto é, quando certos teoremas são provados). Nesse sentido, o que determina a objetividade do processo de medição, "o que é invariante e, portanto, não é uma questão de convenção, é a estrutura relacional empírica e suas propriedades empíricas, algumas das quais são formuladas como axiomas" (Krantz et al, 1971, p. 13).

Assim, a abordagem recusa RMC, mas permanece fundacionalista sobre os processos de medida. O ponto controverso aqui consiste na pergunta sobre o que é, de fato, esse fundamento. Por um lado, a abordagem

33 Essa abordagem adquiriu maturidade na já citada Teoria Representacional da Medição (cf. Díez, 1996a, 1996b e Michell, 1993, 1999).

${ }^{34}$ Nesse contexto, homomorfismo é uma função que preserva relações estruturais; não sendo necessário que hajam isomorfismos. 
representacionalista possui a pretensão de afirmar o caráter empírico das representações numéricas, atribuindo às regularidades observadas e representadas através de axiomas o papel de fundamentar o restante do processo. Como afirma Kyburg (1997) o antirrealismo de viés representacionalista entende que "uma representação numérica se destina a capturar apenas certas regularidades qualitativas [...] relações entre os objetos" (Kyburg, 1997, p. 406, tradução nossa). Por outro lado, o caráter empírico dessa abordagem tem sido criticado. Como a abordagem não trata dos problemas filosóficos que emergem da postulação de que regularidades são observadas, como por exemplo a exigência de conhecimentos teóricos para a construção e preparação dos instrumentos, autores como Heilmann (2015) afirmam que ela é melhor entendida enquanto uma ferramenta formal, cuja aplicação resulta em um corpo teórico que consiste em uma coleção de teoremas. Desse outro ponto de vista, os axiomas e a estrutura relacional qualitativa não representam relações (atuais) entre objetos, mas relações idealizadas e conceituais. Essa interpretação da abordagem representacional torna claro que os fundamentos do seu processo não podem ser vistas enquanto relações empíricas, pois: "nós devemos idealizar as relações observáveis [...] com base nessas relações idealizadas, podemos definir classes de equivalências no domínio [...] nosso realismo consiste na suposição de que essas classes existem" (Kyburg, 1997, p. 397, tradução nossa).

Muito por conta dessas considerações falamos em uma "área cinzenta" nesse artigo. Dependendo de como interpretamos o que é o cerne fundacionalista e quais os comprometimentos ontológicos da abordagem representacionalista, encontramos elementos que 
Perspectiva Filosófica, vol. 45, n. 1, 2018

permitiriam a caracterizar como diferentes posturas (antir)realistas sobre as medições científicas. Por exemplo, são diferentes explanações sobre os comprometimentos ontológicos da abordagem representacionalista que originam os realismos defendidos Mundy (1987), Swoyer(1987), mas também por Michell (1993, 1999, 2004, 2005); bem como o "realismo modesto" afirmado por Kyburg: "um realismo modesto aceitando a existência independente de magnitudes enquanto valores para quantidades a partir da visão representacionalista" (1997, p. 407, tradução nossa);

Do nosso ponto de vista, essa variedade é explicada quando observamos, como Heilmann (2015) o faz, que o núcleo da abordagem representacionalista consiste em uma ferramenta, um método para vincular relações numéricas com relações não numéricas. Nesse sentido, o cerne dessa abordagem consiste na afirmação de que os outcomes das medições são representações apropriadas se há um homomorfismo entre uma estrutura qualitativa e uma estrutura quantitativa. Porém, aquilo que fundamenta essa ferramenta extrapola o corpo teórico da abordagem (a coleção de teoremas que ela origina). Em síntese, sabemos que a abordagem afirma que há um fundamento para o processo de representação numérica e que esses devem ser "relações", mas não há unanimidade sobre o que são essas relações, nem sobre o que elas significam em um processo de medição.

Contudo, a não ser que a abordagem representacionalista seja complementada por análises que discutam seus comprometimentos ontológicos e argumentem que a ferramenta é explicada e possui melhor desempenho à luz de uma forma de RMC, a mesma não implica na defesa do RMC. Assim, ela é aqui considerada 
antirrealista por recusar II) com a afirmação de que os outcomes das medições são representações que dependem tanto de relações (conceituais ou observáveis), quanto do procedimento que permite o seu acesso.

\section{4 - Considerações finais}

O presente artigo possuiu por objetivo oferecer uma taxonomia para os diferentes tipos de (antir)realismos no contexto da filosofia da medição. Para isso, construímos uma visão intuitiva e processual do que são as medições, relacionando esse processo com uma intuição específica sobre o que signifique afirmar que ele é objetivo. Como resultado de nossa análise, encontramos que o realismo sobre as medições científicas, o RMC, é uma tese específica sobre como essa objetividade é garantida, que pode ser complementada por considerações metodológicas e ontológicas, formando os $\mathrm{RMC}+\mathrm{x}$. Como há diferentes maneiras de recusar o RMC, encontramos um tipo de antirrealismo que recusa sua tese em geral recusando que as medições possam ser objetivas, o ARMC; mas outros posicionamentos que atentam para a especificidade do RMC. Nesse último caso, distinguimos entre posturas que construíram teorizações sobre a objetividade dos processos de medições a partir de vieses fundacionalistas, o AFMC, ou coerentistas, o ACMC.

Em síntese, essas distinções formam uma taxonomia inicial sobre como a questão do realismo adentra as discussões em filosofia da medição. Se ela fez sentido até aqui, a partir do exposto podemos não só aprofundar elementos específicos dessa discussão, mas principalmente explorar as relações de compatibilidade entre os posicionamentos aqui apresentados com aqueles que encontramos na discussão do RC, constituindo um ponto de partida frutífero para trabalhos futuros. 
Perspectiva Filosófica, vol. 45, n. 1, 2018

\section{Referências bibliográficas}

BYERLY, C. LAZARA, V. Realist Foundations of Measurement. Philosophy of Science, v. 40, p. 10-28, 1973.

CAMPBELL, Norman. Physics: The Elements. London: Cambridge University Press, 1920.

CARTWRIGHT, Nancy. The Dappled World: A Study of the Boundaries of Science. Cambridge: Cambridge University Press, 1999.

CARTWRIGHT, Nancy. CHANG, Hasok. Measurement. In: CURD, M. PSILLOS, S. (ed). The Routledge Companion to Philosophy of Science. 2nd ed. New York: Routledge, p. 411 - 419, 2013.

CHAKRAVARTTY, Anjan. Scientific Realism. In: ZALTA, Edward. The Stanford Encyclopedia of Philosophy, 2017. Disponível em: <plato.stanford.edu/archives/sum2017/entries/scientificrealism/>. Acesso em: fev. 2019.

DÍEZ, José. A Hundred Years of Numbers. An Historical Introduction to Measurement Theory 1887-1990-Part 1. Studies in History and Philosophy of Science, v. 28, pp. 167-185, 1997a.

A Hundred Years of Numbers. An Historical Introduction to Measurement Theory 1887-1990-Part 2. Studies in History and Philosophy of Science, v. 28, pp. 237-265, 1997b.

DUNWAY, Billy. Realism and objectivity. In: McPHERSON, T. PLUNKETT, D (eds). The Routledge Handbook of Metaethics. New York: Routledge, p. 135-150, 2018.

FINKELSTEIN, L. Introductory Article. Journal of Physics, v. 10, p. 566-572, 1977. 
Perspectiva Filosófica, vol. 45, n. 1, 2018

FRIGERIO, Aldo. GIORDANI, Alessandro. MARI, Luca. Outline of a general model of measurement, Synthese, n. 175 v. 2, p. 123-149. 2010.

KRANTZ, David. LUCE, Duncan. SUPPES, Patrick. TVERSKY, Amos. Foundations of Measurement Vol 1: Additive and Polynomial Representations. London: Academic Press, 1971.

KYBURG, Henry. Quantities, Magnitudes, and Numbers. Philosophy of Science, v. 64, p. 377-410, 1977.

HAACK, Susan. Science is neither sacred nor a confidence trick. Foundations of Science, v. 3, 1995, p. 323-335.

HACKING, Ian. Experimentation and Scientific Realism. Philosophical Topics, v. 13, p. 71-87, 1982.

HEILMANN, Conrad. A New Interpretation of the Representational Theory of Measurement. Philosophy of Science, v. 82, n. 5, p. 787-797, 2015.

HELMHOLTZ, Hermann. Numbering and Measuring from an Epistemological Viewpoint. In: COHEN, R; ELKANA, Y (eds). Hermann von Helmholtz epistemological writings. Boston: D. REIDEL PUBLISHING COMPANY, p. 72-102, 1921.

MARI, Luca. CARBONEB, Paolo. GIORDANIC, A. PETRID, D. A structural interpretation of measurement and some related epistemological Studies in History and Philosophy of Science, v. 65-66, 2017, p. 46-56.

MICHELL, Joel. History and philosophy of measurement: A realist view. Artigo apresentado no evento: Proceedings of the 10th IMEKO TC7 International symposium on advances of measurement science, 2004. Disponível em: <https://web.archive.org/web/20160409214805/http://www. imeko.org/publications/tc7-2004/IMEKO-TC7-2004128.pdf>. Acesso em: dez., 2018. 
- Making the Representational theory of measurement. In: MICHELL, Joel. Measurement in psychology: A critical history of a methodological concept. Cambridge: Cambridge University Press, p. 109-139, 1999. . The Origins of the Representational Theory of Measurement: Helmholtz, Hölder, and Russell. Studies in History and Philosophy of Science, v. 24, n. 2, pp. 185-206, 1993.

Measurement, v. 38, p. 285-294, 2005.

RIORDAN, Sally. The Objectivity of Scientific Measures. Studies in History and Philosophy of Science, v. 50, p. 3847, 2015.

STEVENS, Stanley. On the Theory of Scales of Measurement. Science, v. 103, p. 677-680, 1946.

SWOYER, C., 1987. The Metaphysics of Measurement: Essays on Measurement in the Social and Physical Sciences. In: FORGE, J (ed). Measurement, Realism and Objectivity. Dordrecht: Reidel, pp. 235-290, 1987.

TAL, Eran. A Model-based Epistemology of Measurement. In: MÖßNER, N. NORDMANN, A. (eds). Reasoning in Measurement. London: Pickering \& Chatto Publishers, $2017 \mathrm{a}$.

TAL, Eran. Measurement in Science. In: ZALTA, E. (ed). The Stanford Encyclopedia of Philosophy, 2017b. Disponível em:

$<$ https://plato.stanford.edu/archives/fall2017/entries/measu rement-science/>. Acesso em: dez., 2017.

- Old and New Problems in Philosophy of Measurement. Philosophy Compass, v.8, 2013, pp. 11591173.

TELLER, Paul. Measurement Accuracy Realism. Artigo apresentado no evento: Foundations of Physics 2013 - The 17th UK and European Meeting on the Foundations of 
Perspectiva Filosófica, vol. 45, n. 1, 2018

Physics. 2013b. Disponível em: <http://philsciarchive.pitt.edu/9740/>. Acesso em: jan. 2018.

. The Concept of Measurement-precision.

Synthese, v. 190, p. 189-202, 2013a.

Van BRAKEL, J. Norms and facts in measurement. Measurement, v. 2, p. 45-51, 1984. 